\title{
Analyzing China's Term Structure of Interest Rates Using VAR and Nelson-Siegel Model
}

\author{
Minjie Ding \\ Peking University, Beijing, China \\ Email: dingminjie@pku.edu.cn
}

How to cite this paper: Ding, M.J. (2020) Analyzing China's Term Structure of Interest Rates Using VAR and Nelson-Siegel Model. Journal of Mathematical Finance, $10,242-254$.

https://doi.org/10.4236/jmf.2020.102015

Received: January 20, 2020

Accepted: May 8, 2020

Published: May 11, 2020

Copyright $\odot 2020$ by author(s) and Scientific Research Publishing Inc. This work is licensed under the Creative Commons Attribution International License (CC BY 4.0).

http://creativecommons.org/licenses/by/4.0/

(c) (i) Open Access

\begin{abstract}
China's bonds market has developed rapidly in recent years. A further study of interest rate term structure is essential. Nelson-Siegel model is widely used to fit interest rate term structure around the world. In this essay, we try to find out whether Nelson-Siegel model is efficiency in China, and which model is most efficient among some typical variants of Nelson-Siegel model. After brief theoretical introduction, we conduct empirical analysis, which contains two sections. In the first session, we focus on fitting Chinese interest rate term structure using Nelson-Siegel model, and fitting efficiency turns out to be pretty good. In the second section, we establish a VAR model with macroeconomic variables to predict parameters in Nelson-Siegel model, and use the combination of VAR and NS model to predict interest rate term structure in 2019 and 2020 respectively. Also, in terms of prediction efficiency, VAR (Macro)-NS model performs better than both VAR-NS model without macroeconomic variables and simple NS model.
\end{abstract}

\section{Keywords}

China’s Term Structure, Nelson-Siegel Model

\section{Introduction}

Interest rate is one of most important variables in the financial market, which can depict the relationship between money supply and money demand. Treasury bonds are regarded as risk-free interest rate for high credit and low risk. Treasury bond yield curve reflects the relationship between bond yield and term to maturity, so it is also known as interest rate term structure. Treasury bond yield curve becomes benchmark for financial products, and it's critical to financial market.

China's treasury bond curve began to form since year 2002. Electronic trea- 
sury bonds were introduced in 2006, which increased the amount of treasury investment. By the end of 2019, total market value of China's bond market reached 97,060 billion RMB, yoy $13.2 \%$, becoming the second largest bond market in the world.

Scholars and investors all over world have shown great interest in treasury bond yield curve, for its important role in financial market. Treasury bond yield curves in different countries present different characters. In China, interest rate used to be regulated, and it's not easy to predict interest rate barely from market prospective. With the progress of interest rate marketization, does China's treasury bond yield curve becomes more easy to predict? Is the prediction convincing? This paper will focus on these questions in following discussion.

This paper uses the method put up by Nelson \& Siegel (1987), to fit treasury bond yield curve. This method is practical for few parameters, and each parameter has economic meanings. Also, this paper follows the research structure by Diebold \& Li (2006). First, the paper fits China's treasury bond yield curve using Nelson-Siegel method and tests its efficiency. After that, the paper establishes VAR (vector auto-regression model) to predict parameters in Nelson-Siegel model. Then, the paper gives the predicted treasury bond yield curve of 2020 .

Compared with relevant researches conducted by scholars and investor, this paper does some new work. First, the paper uses yield curve data from CCDC (China Central Depository \& Clearing Co., Ltd., http://www.ccdc.com.cn/ccdc/en/index.shtml) as raw data, while other researchers usually use specific traded treasury bond in market. CCDC has already filtered market noise when CCDC calculates yield rate. Also, regulatory department has assigned CCDC's treasury bond yield curve as pricing benchmark. For example, China Banking Regulatory Commission requires commercial banks to use CCDC's treasury bond yield rate as benchmark of risk management. Second, based on trade experience in Chinese bond market, the paper introduced several typical macroeconomic variables into dynamic Nelson-Siegel model, and established VAR(Macro)-NS model. Third, in order to test the efficiency of VAR(Macro)-NS model, this paper introduces a VAR-NS model and a NS model. In addition, although there are already scholars around the world discussing the effect from macro variables on term structure, the importance of this topic is not highlighted in China, partly because of long-term regulated interest rate market and difficulty to get raw market data. This paper is first to fully exam effect from macro variables on term structure using most proper market data in China market.

The remainder of this paper is as follows. Section 2 shows literature review of Nelson-Siegel model, including recent research done by Chinese scholars. Section 3 gives brief introduction to Nelson-Siegel model. Section 4 describes how we estimate the level, slope and curvature factors of the yield curve, and we test whether Nelson-Siegel model can fit Chinese treasury bond yield curve well. Section 5 examines predictive ability and gives our predicted yield curve based 
on VAR(Macro)-NS model.

\section{Literature Review}

As scholars and investors use yield term structure more frequently, more relative models come up.

Nelson-Siegel model was first proposed by Nelson and Siegel (1987). This model is widely favored, for its intuitive economic explanation, less parameters to estimate and good fitting performance. However, Nelson-Siegel model fails to produce a multi-peak yield curve. Svensson (1994) added an exponential polynomial to solve the problem, and got Nelson-Siegel-Svensson model. Although Nelson-Siegel-Svensson model can generate a multi-peak yield curve, too many parameters makes it too sensitive to initial value.

Nelson-Siegel models are widely used both by academic research and government policy. According to report from BIS (2005) [1], central banks of Belgium, Finland, France, Italy and Span use Nelson-Siegel models to estimate yield term structure, and centrals banks of US, Canada, UK, German and Switzerland choose Nelson-Siegel-Svensson model.

Dynamic Nelson-Siegel model was first set up by Diebold and Li (2006) [2]. They assumed that factors of NS model follow AR (1) process. Christensen, Diebold and Rudebusch (2009) [3] consider no-arbitrage restriction in DNS model, which is called AFDNS model. Koopman, Malle and Van (2010) [4] add GARCH process into DNS model.

In China, Shen (2010) [5] applied DNS model to Chinese inter-bank market. Wang (2010) studied no-arbitrage DNS model and found the slope factor has closer correlation with macroeconomics variables. Shen and Shuai (2017) [6] decreased parameter numbers in Lengwiler and Lenz's (2010) [7] model, they introduced VARMA $(1,1)$ into DNS model and ensure the independence of impulse from factors.

In addition, many scholars and investors began to explore the relationship between interest rate and macroeconomic variables.

Ang and Piazzesi (2003) [8] added macroeconomic variables to interest rate model. They got a better predictability, and found that $85 \%$ of yield volatility could be explained by macroeconomic variables. Based on this work, Bikbov and Chernov (2010) [9] proposed a model without restrictions on the number of latent variables. Also, discussion about forecast horizons came up, Hordahl (2006) and Moench (2008) [10] found model with macro variables had better forecasting performance only for longer prediction horizons, while de Pooter (2010) and Altavilla (2014) outperformed random walk benchmarks only for short-term maturities at shorter horizons. Coroneo (2016) [11] added backward-looking macroeconomic variables into term structure model, and his model performed better than random walk forecasts from 3 to 24 months ahead. It is argued that macro variables is useful particularly in certain years, such as in recessionary periods. This argument is supported by evidence provided by Guidolin and Pedio's 
(2019) [12], whose models with monetary policy variables significantly increased predictive ability during 2008 financial crisis.

Interest rate in China was partially regulated until recent years. As a result, Chinese scholar didn't pay much attention to macroeconomic variables and yield term structure before 2010. In recent years, relevant studies came up, as interest rate realized liberalization. Zeng and Niu (2013) became first Chinese scholar who got term structure of real interest rate and inflation rate, using no-arbitrage models. They also found that inflation rate affected more on short-term yields, while real interest rate affected more on long-term yields. Qiang and Hou (2018) [13] built an affine interest rate model based on benchmark interest rate, market liquidity and risk premium, which explained the term structure well. They found that benchmark interest rate is determinant to other term yields, and market liquidity influence more on short-term yields, while risk premium affects more on long-term yields.

\section{Brief Introduction to Nelson-Siegel Model}

In 1987, Nelson and Siegel first use Nelson-Siegel model to fit yield curve, and the model is as follows.

$$
y(\tau)=\beta_{1}+\beta_{2} *\left(\frac{1-\mathrm{e}^{-\lambda \tau}}{\lambda \tau}\right)+\beta_{3} *\left(\frac{1-\mathrm{e}^{-\lambda \tau}}{\lambda \tau}-\mathrm{e}^{-\lambda \tau}\right)
$$

Diebold and Li said that yield rate should change with time, and put forward Dynamic Nelson-Siegel model, and the model is as follows.

$$
y_{t}(\tau)=\beta_{1 t}+\beta_{2 t} *\left(\frac{1-\mathrm{e}^{-\lambda \tau}}{\lambda \tau}\right)+\beta_{3 t} *\left(\frac{1-\mathrm{e}^{-\lambda \tau}}{\lambda \tau}-\mathrm{e}^{-\lambda \tau}\right)
$$

In the formula above, $t$ represents a specific time point. $\tau$ represents time to maturity. $y_{t}(\tau)$ represents the yield rate of a treasury bond with $\tau$ to maturity at time $t$. $\beta_{1 t}, \beta_{2 t}, \beta_{3 t}$ and $\lambda$ are parameters to be estimate.

- As $\tau$ approaches infinity, we get $\lim _{\tau \rightarrow \infty} y_{t}(\tau)=\beta_{1 t}$. So $\beta_{1 t}$ is regarded as horizontal parameter. Change of $\beta_{1 t}$ will change the overall yield rate of a treasury bond.

- As $\tau$ approaches zero, we get $\lim y_{t}(\tau)=\beta_{1 t}+\beta_{2 t}$. After transposition, we get $\beta_{2 t}=y_{t}(0)-\beta_{1 t}=y_{t}(0)-y_{t}(\infty)$. So $\beta_{2 t}$ is regarded as slope parameter.

- When $\tau$ is a normal number around 60, which means the treasury bond has 5 years (60 months) to maturity, we approximately get $y_{t}(60)=\beta_{1 t}+0.5 * \beta_{2 t}+0.5 * \beta_{3 t}$. After transposition, we get $\beta_{3 t}=\left(y_{t}(0)-y_{t}(\tau)\right)-\left(y_{t}(\tau)-y_{t}(\infty)\right)$. So $\beta_{3 t}$ is usually regarded as curvature parameter.

The right side of formula divides the yield rate into three parts. The first item is $\beta_{1 t}$, which affects long-term yield rate. We can call it long-term factor. The second item is $\frac{1-\mathrm{e}^{-\lambda \tau}}{\lambda \tau}$, when $\tau$ approaches infinity, this item approaches ze- 
ro, which means this item affect yield rate more in short term, as shown in Figure 1 . We can call it short-term factor. The third item is $\frac{1-\mathrm{e}^{-\lambda \tau}}{\lambda \tau}-\mathrm{e}^{-\lambda \tau}$, as $\tau$ approaches infinity or zero, the third item approaches zero, which means its effect is around medium-term, as shown in Figure 2, so we can call it medium-term factor. So all factors that affect yield curve can be divided into these three group, as shown in Figure 3.

$\lambda$ is usually given previous to other work. On the one side, $\lambda$ determines when $\frac{1-\mathrm{e}^{-\lambda \tau}}{\lambda \tau}-\mathrm{e}^{-\lambda \tau}$ will reach largest value. On the other side, $\lambda$ determines the speed $\frac{1-\mathrm{e}^{-\lambda \tau}}{\lambda \tau}$ decreases with time. Diebold and $\operatorname{Li}(2006)$ take $\lambda=0.0609$, which means medium factor reaches largest in $30^{\text {th }}$ month.

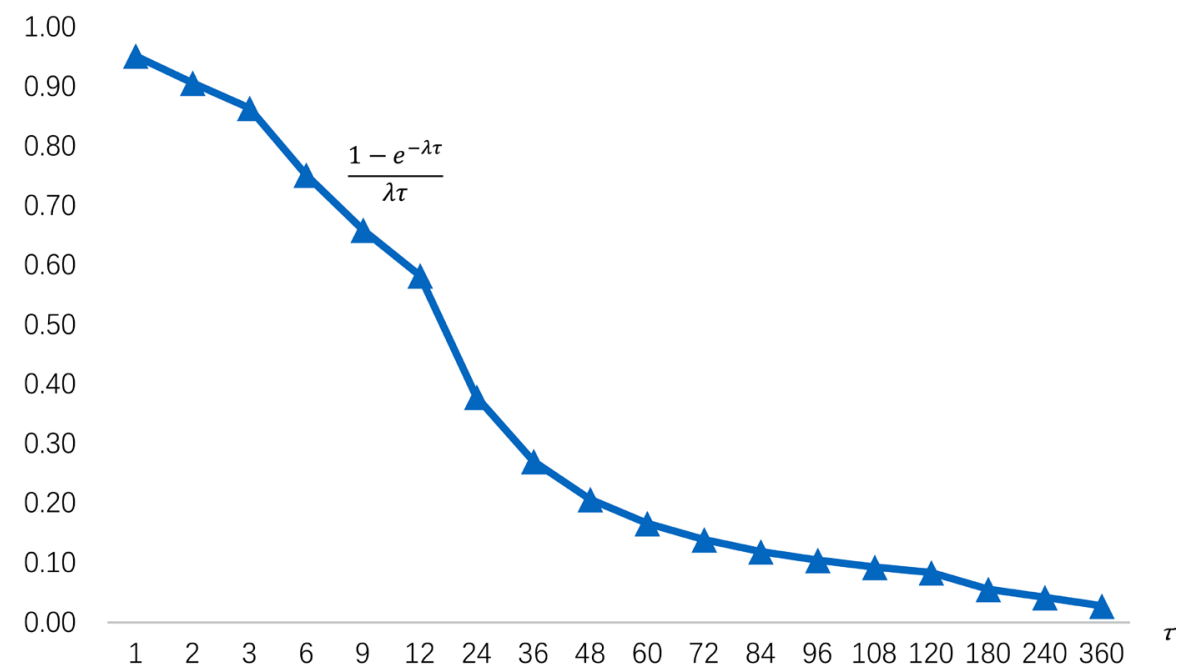

Figure 1. $\frac{1-\mathrm{e}^{-\lambda \tau}}{\lambda \tau}$ trend (assuming $\lambda=0.1$ ).

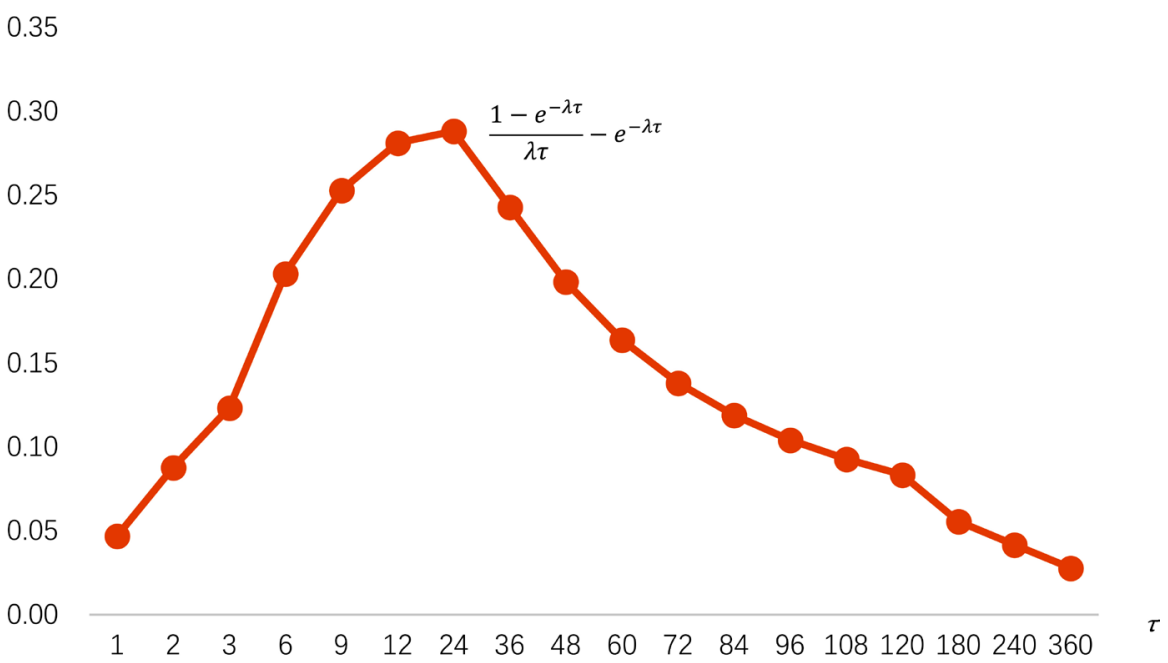

Figure 2. $\frac{1-\mathrm{e}^{-\lambda \tau}}{\lambda \tau}-\mathrm{e}^{-\lambda \tau}$ trend (assuming $\lambda=0.1$ ). 


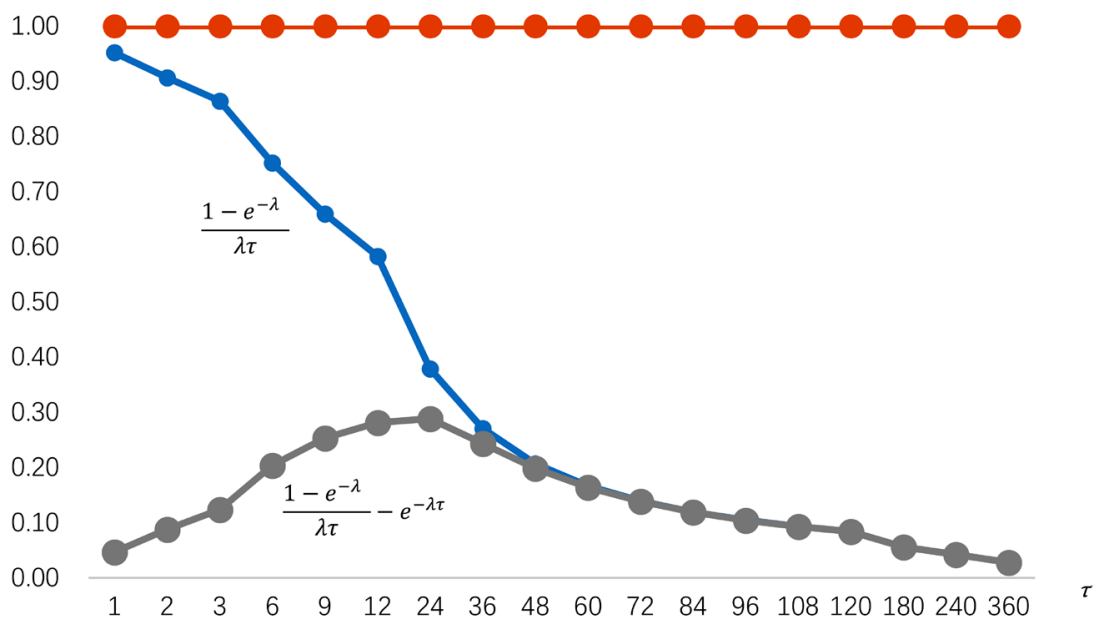

Figure 3. Deposition of factors that affect yield rate.

\section{Can Nelson-Siegel Model Fit Chinese Treasury Bond Yield Curve Well?}

\subsection{Search for Best Value of $\lambda$}

Considering that $\lambda$ has an influence on the fitting effect, we should first choose an optimal $\lambda$. Here we take this method. The first step is choose a certain $\lambda$, then we calculate $\beta_{1 t}, \beta_{2 t}, \beta_{3 t}$ base on this given $\lambda$. After that, we use $\beta_{1 t}, \beta_{2 t}, \beta_{3 t}$ to get fitting value and their estimation error. By now, we get an estimation error, based on a given $\lambda$. Now, we tried each $\lambda$ between 0.005 and 0.070 , with interval of 0.001 .

We take 204 monthly treasury bond yield curve from year 2002 to year 2018 as our sample. Also, considering that bonds with more than 10 years to maturity may have deviation because of lack of liquidity, we choose bonds whose term to maturity is between 1 month and 10 years.

After all 65 trials, we find that, the estimation error is least when $\lambda$ is 0.030 , which means the medium factor become largest in around $4^{\text {th }}$ and $5^{\text {th }}$ year, as shown in Figure 4.

\subsection{Fitting Effect of Nelson-Siegel Model on Chinese Treasury Bond}

We take 204 monthly treasury bond yield curve from year 2002 to year 2018 as our raw data. Firstly, we use Nelson-Siegel model to do monthly regression, and get $\beta_{1 t}, \beta_{2 t}, \beta_{3 t}$ for each month and average $\beta_{1 t}, \beta_{2 t}, \beta_{3 t}$ during the sample period. Then we use the average $\beta_{1 t}, \beta_{2 t}, \beta_{3 t}$ to get predicted yield rate for each maturity, and compare them with real yield rate. The table below shows the result. As we can see in Table 1, the prediction error is all between $0 \sim 5$ basis point, which demonstrate that Nelson-Siegel model can give an excellent prediction on Chinese treasury bond yield curve.

\subsection{Test of Meaning of Parameters}

As we discussed above, $\beta_{1 t}$ represents horizontal parameter, $\beta_{2 t}$ represents 
slope parameter and $\beta_{3 t}$ represents curvature parameter in Nelson-Siegel model. While in real trading market, we can use yield rate of bonds with 10 year to maturity to represent horizontal level of yield curve. Also, real slope is often approximately calculated by substracting 1-year yield rate from 10-year yield rate. Moreover, we can use "(10-year yield rate - 10-year yield rate) - (5-year yield rate - 1-year yield rate)" to approximately represent real curvature. Our calculation result is in Table 2. We find that the correlation between $\beta_{1 t}$ and real horizontal level is 0.866 , the correlation between $\beta_{2 t}$ and real slope is -0.964 and the correlation between $\beta_{3 t}$ and real curvature -0.554 . These three parameters all have close relationship with real observed values. Figure 5, Figure 6 and Figure 7 show the trends of three relationships mentioned above, which further manifest the close correlation.

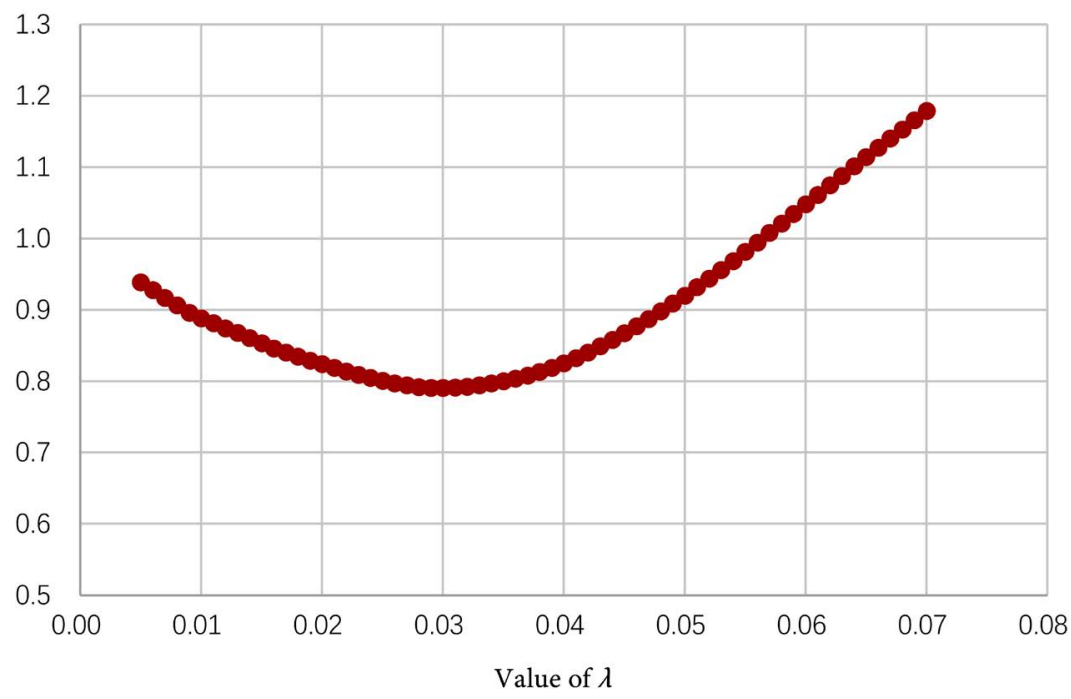

Figure 4. Average absolute fitting error from Nelson-Siegel model with different $\lambda$.

Table 1. Real yield rate vs. fitted yield rate in 2002-2018.

\begin{tabular}{cccccccccccccccccccccc}
\hline Time to maturity $\tau$ (month) & 2 & 3 & 6 & 9 & 12 & 24 & 36 & 48 & 60 & 72 & 84 & 96 & 108 & 120 \\
\hline $\begin{array}{c}\text { Average historical yield rate } \\
(\%)\end{array}$ & 2.3806 & 2.4132 & 2.4810 & 2.5256 & 2.5745 & 2.7624 & 2.9253 & 3.0673 & 3.1769 & 3.3090 & 3.3960 & 3.4719 & 3.5313 & 3.5837 \\
Fitted yield rate (\%) & 2.3767 & 2.3952 & 2.4498 & 2.5030 & 2.5547 & 2.7470 & 2.9166 & 3.0650 & 3.1941 & 3.3063 & 3.4037 & 3.4882 & 3.5617 & 3.6258 \\
Predicted error (BP) & -0.38 & -1.80 & -3.12 & -2.26 & -1.98 & -1.54 & -0.87 & -0.23 & 1.73 & -0.27 & 0.77 & 1.63 & 3.05 & 4.21 \\
\hline
\end{tabular}

Table 2. Correlation between estimated parameters and real term structure.

\begin{tabular}{cccc}
\hline $\begin{array}{c}\text { Observed height } \\
\text { of yield curve }\end{array}$ & $\begin{array}{c}\text { Observed slope } \\
\text { of yield curve }\end{array}$ & Observed curvature of yield curve \\
\hline Yield rate (10) & $\begin{array}{c}\text { Yield rate (10) minus } \\
\text { yield rate (1) }\end{array}$ & $\begin{array}{c}\text { Yield rate (10) plus yield rate (1), minus } \\
\text { two times yield rate (5) }\end{array}$ \\
$\beta_{1 t}$ & $\mathbf{0 . 8 6 6}$ & 0.398 & -0.010 \\
$\beta_{2 t}$ & -0.074 & -0.964 & 0.147 \\
$\beta_{3 t}$ & 0.341 & 0.031 & -0.554
\end{tabular}

Note: Yield rate $(\mathrm{x})$ means the yield rate of treasury bond with $\mathrm{x}$ years to maturity. 


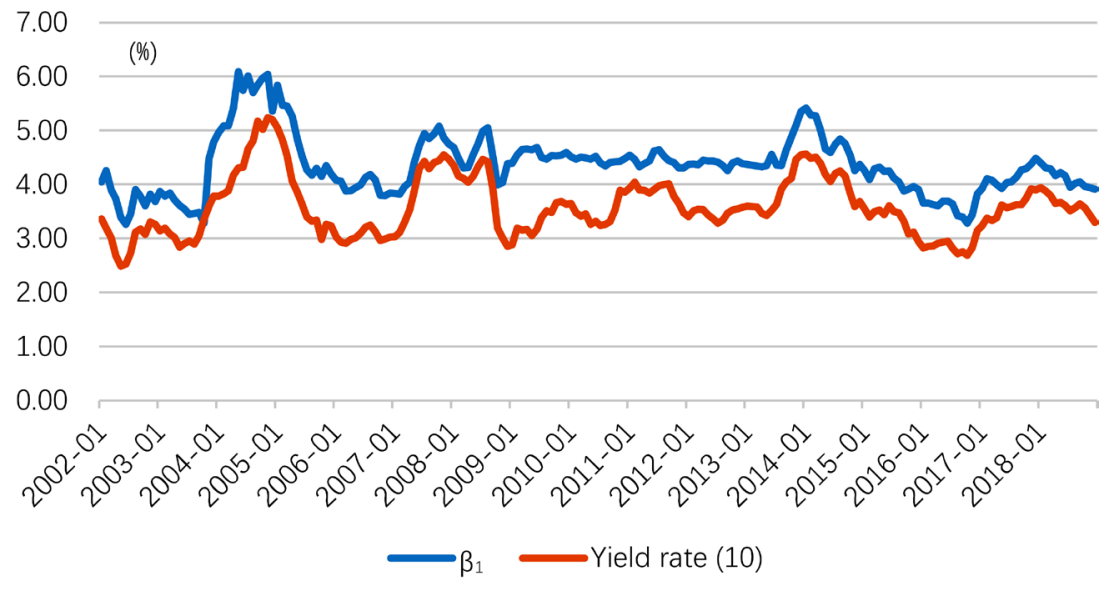

Note: Yield rate (10) mean the yield rate of treasury bond with 10 years to maturity.

Figure 5. Trends of $\beta_{1 t}$ and yield rate (10).

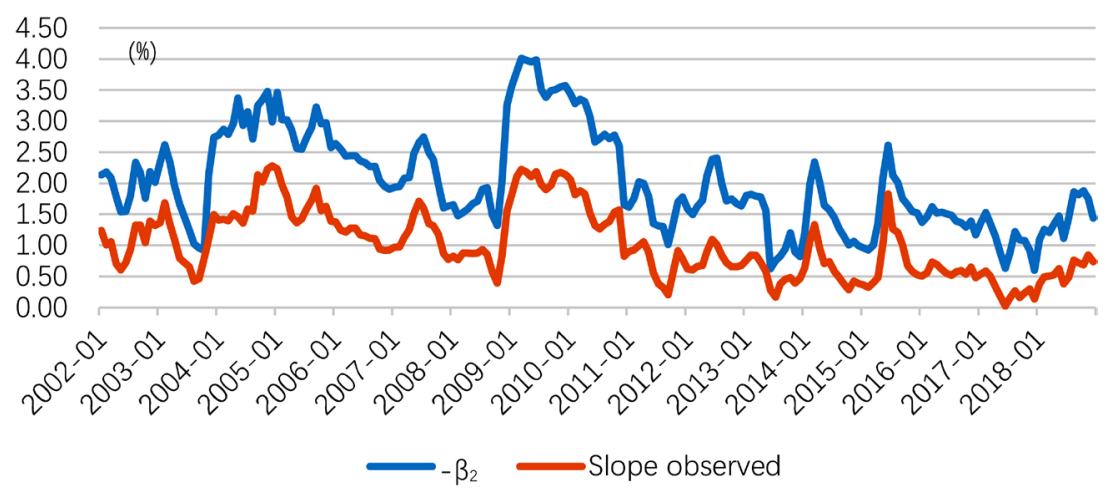

Note: Slope of yield curve observed is approximately calculate by yield rate of 10 -year treasury bond minus that of 1-year treasury bond, that is yield rate (10)-yield rate (1).

Figure 6. Trends of $-\beta_{2 t}$ and real slope of yield curve.

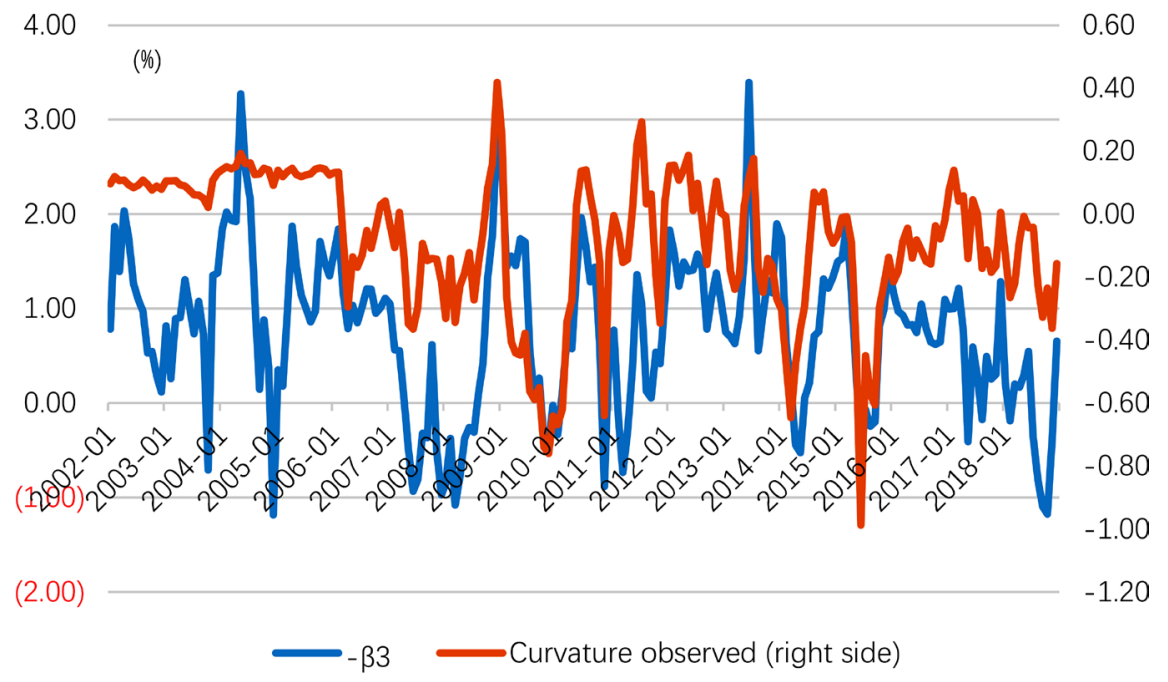

Note: Curvature of yield curve is approximately calculate by yield rate of 10 -year treasury bond minus plus that of 1 year treasury bond, subtracted by two time yield rate of 5 -year treasury bond, that is yield rate (10) + yield rate (1)-2* yield rate(5).

Figure 7. Trends of $-\beta_{3 t}$ and real curvature of yield curve. 


\subsection{Macroeconomic Variables Affecting Parameters}

There are many factors which affect the yield rate of bonds. In Chinese bonds market, these factors can be mainly divided into three categories, which are economic growth, inflation and market liquidity. Industrial Added Value in China is a monthly published data, and these industries contribute a lot to China's GDP, so we choose IAV (Industry Added Value) to represent economic growth. And we choose CPI to represent inflation, and choose growth of M2 to represent market liquidity.

Now, we try to find correlation among $\beta_{1 t}, \beta_{2 t}, \beta_{3 t}$ and macroeconomic variables. The results is shown in Table 3, $\beta_{1 t}$ has a close correlation with CPI and IAV, $\beta_{2 t}$ is correlated with IAV most, and $\beta_{3 t}$ is correlated with CPI most.

\section{Predict Yield Curve Based on VAR(Macro)-NS Model}

\subsection{Variable Selection and Stationarity Test}

The key to predict yield curve is to getting value of parameter $\beta_{1 t}, \beta_{2 t}, \beta_{3 t}$. We take VAR, or Vector Auto-regression Model, into consideration. As we discussed above, macroeconomics variables, including growth rate of Industry Added Value, CPI and M2, are closely related with parameter $\beta_{1 t}, \beta_{2 t}, \beta_{3 t}$. So, our model will include these macroeconomic variables.

The sample is monthly yield curve data from Jan 2002 to Dec 2018. We will use the model predict yield curve of 2019. Then we compare the predicted 2019 yield curve with observed yield curve.

As shown in Table 4 , our variables, including $\beta_{1 t}, \beta_{2 t}, \beta_{3 t}$, IAV (Industry Added Value), CPI and M2, all pass ADF stationarity test.

Table 3. Correlation between $\beta_{1 t}, \beta_{2 t}, \beta_{3 t}$ and macroeconomic variables in 2002-2018.

\begin{tabular}{ccccccc}
\hline & CPI & Ind & M2 & CPI (lag) & Ind (lag) & M2 (lag) \\
\hline$\beta_{1 t}$ & $\mathbf{0 . 4 2}$ & $\mathbf{0 . 3 7}$ & 0.31 & $\mathbf{0 . 4 4 ( + 2 )}$ & $\mathbf{0 . 4 2 ( + 7 )}$ & $0.52(+10)$ \\
$\beta_{2 t}$ & 0.17 & -0.47 & -0.59 & 0.17 & $-0.52(+10)$ & -0.59 \\
$\beta_{3 t}$ & $\mathbf{0 . 3 1}$ & -0.17 & -0.11 & $\mathbf{0 . 3 7}(-2)$ & $-0.20(+4)$ & $-0.16(+6)$ \\
\hline
\end{tabular}

Note: “ $+x$ ” above means that macroeconomic variables leads $\mathrm{x}$ months ahead of parameters.

Table 4. ADF test of three parameters and macroeconomic variables.

\begin{tabular}{ccc}
\hline Parameters and variables & Dickey-Fuller statistics & P value \\
\hline$\beta_{1 t}$ & -3.5005 & 0.04379 \\
$\beta_{2 t}$ & -3.4625 & 0.04739 \\
$\beta_{3 t}$ & -4.4305 & $<0.01$ \\
IAV & -4.8190 & $<0.01$ \\
CPI & -3.7951 & 0.02026 \\
$\Delta \mathrm{M} 2$ & -4.5536 & $<0.01$ \\
\hline
\end{tabular}




\subsection{Discussion on Prediction Result}

After processing data, we get the result of VAR(macro)-NS model as follows.

$$
\begin{aligned}
& \beta_{1 t}=1.063 * \beta_{1, t-1}-0.005 * \beta_{1, t-2}-0.124 * \beta_{1, t-3}-0.168 * \beta_{1, t-4} \\
& +0.123 * \beta_{1, t-5}+0.264 * \beta_{1, t-6}+0.401 * \beta_{1, t-7}+0.252 * \beta_{1, t-8} \\
& -0.201 * \beta_{1, t-9}+0.246 * \beta_{1, t-10}-0.207 * \beta_{1, t-11}+0.024 * \beta_{1, t-12} \\
& +0.063 * \mathrm{CPI}_{t-1}+0.056 * \mathrm{CPI}_{t-2}+0.001 * \mathrm{CPI}_{t-3}-0.005 * \mathrm{CPI}_{t-4} \\
& -0.001 * \mathrm{CPI}_{t-5}+0.028 * \mathrm{CPI}_{t-6}-0.035 * \mathrm{CPI}_{t-7}-0.064 * \mathrm{CPI}_{t-8} \\
& +0.065 * \mathrm{CPI}_{t-9}+0.053 * \mathrm{CPI}_{t-10}-0.030 * \mathrm{CPI}_{t-11} \\
& -0.001 * \mathrm{CPI}_{t-12}-0.005 * \mathrm{IAV}_{t-1}-0.012 * \mathrm{IAV}_{t-2} \\
& +0.058 * \mathrm{IAV}_{t-3}-0.007 * \mathrm{IAV}_{t-4}+0.007 * \mathrm{IAV}_{t-5} \\
& -0.091 * \mathrm{IAV}_{t-6}-0.006 * \mathrm{IAV}_{t-7}-0.068 * \mathrm{IAV}_{t-8} \\
& +0.132 * \mathrm{IAV}_{t-9}-0.135 * \mathrm{IAV}_{t-10}-0.048 * \mathrm{IAV}_{t-11} \\
& +0.046 * \mathrm{IAV}_{t-12}+0.506 \\
& R^{2}=0.926 \\
& \beta_{2 t}=1.160 * \beta_{2, t-1}-0.280 * \beta_{2, t-2}-0.049 * \beta_{2, t-3}+0.038 * \beta_{2, t-4} \\
& +0.024 * \beta_{2, t-5}-0.083 * \mathrm{M} 2_{t-1}+0.007 * \mathrm{M} 2_{t-2}+0.136 * \mathrm{M} 2_{t-3} \\
& -0.031 * \mathrm{M} 2_{t-4}-0.038 * \mathrm{M} 2_{t-5}-0.063 \\
& R^{2}=0.886 \\
& \beta_{3 t}=0.857 * \beta_{3, t-1}-0.212 * \beta_{3, t-2}+0.176 * \beta_{3, t-3}-0.204 * \beta_{3, t-4} \\
& +0.038 * \beta_{3, t-5}+0.211 * \beta_{3, t-6}-0.274 * \beta_{3, t-7}+0.138 * \beta_{3, t-8} \\
& -0.116 * \beta_{3, t-9}+0.057 * \beta_{3, t-10}-0.012 * \beta_{3, t-11}+0.077 * \beta_{3, t-12} \\
& +0.066 * \mathrm{CPI}_{t-1}+0.007 * \mathrm{CPI}_{t-2}-0.016 * \mathrm{CPI}_{t-3} \\
& +0.005 * \mathrm{CPI}_{t-4}+0.054 * \mathrm{CPI}_{t-5}-0.055 * \mathrm{CPI}_{t-6} \\
& -0.080 * \mathrm{CPI}_{t-7}+0.003 * \mathrm{CPI}_{t-8}+0.112 * \mathrm{CPI}_{t-9} \\
& -0.195 * \mathrm{CPI}_{t-10}+0.066 * \mathrm{CPI}_{t-11}+0.056 * \mathrm{CPI}_{t-12}-0.246 \\
& R^{2}=0.632
\end{aligned}
$$

Using model parameters above, we predict yield curve of 2019 based on VAR(Macro)-NS model. Then we compare the real yield curve with the predicted yield curve. The two yield curve is very close and absolute prediction error is only 13 basis point. Detailed prediction result is in Table 5 .

Table 5. Prediction of 2019 yield curve based on VAR(Macro)-NS model using monthly data from 2002 to 2018.

\begin{tabular}{ccccccccccc}
\hline$\tau$ & 1 & 2 & 3 & 6 & 9 & 12 & 24 & 36 & 48 \\
Predicted yield rate & 2.349 & 2.366 & 2.383 & 2.433 & 2.480 & 2.525 & 2.688 & 2.825 & 2.940 \\
Absolute prediction error & 0.240 & 0.168 & 0.138 & 0.112 & 0.125 & 0.135 & 0.104 & 0.099 & 0.103 \\
$\tau$ & 60 & 72 & 84 & 96 & 108 & 120 & 180 & 240 & 360 \\
Predicted yield rate & 3.037 & 3.119 & 3.189 & 3.249 & 3.300 & 3.344 & 3.493 & 3.574 & 3.658 \\
Absolute prediction error & 0.112 & 0.117 & 0.106 & 0.115 & 0.147 & 0.177 & 0.097 & 0.106 & 0.136 \\
\hline
\end{tabular}


In order to evaluate the efficiency of VAR(Macro)-NS model, we introduce simple NS model and VAR-NS model without macroeconomic variables. The average absolute prediction error of simple NS model is 22 basis point, while VAR-NS model generate absolute prediction error of 15 basis point. Both of them give a larger error than VAR(Macro)-NS model. Detailed prediction result is in Table 6 and Table 7.

\subsection{Predict Yield Curve of $\mathbf{2 0 2 0}$}

Based on monthly yield curve from year 2002 to year 2019 in China, we establish VAR(Macro)-NS model. Then we calculate parameters $\beta_{1 t}, \beta_{2 t}, \beta_{3 t}$ in the model and use this model to produce predicted yield curve of 2020 . The value of predicted macroeconomic variables comes from Wind's prediction, a financial platform widely known as "Chinese Bloomberg". Our predictions are shown in Table 8, Table 9 and Figure 8.

Compaing the treasury bond yield curve of 2018, 2019 and estimated 2020, we find that:

1) Yield curve of 2020 lies almost between curve 2018 and curve 2019. This can be explained by the fact that average $\beta_{1,2020}$ is between $\beta_{1,2018}$ and $\beta_{1,2019}$, which can be further explained by the factor that growth rate of 2020 Industrial Added Value is among that of 2018 and 2019.

2) Yield curve 2020 is more flat than 2018 and 2019, which results from average $\beta_{1,2020}$ is smaller than $\beta_{1,2018}$ and $\beta_{1,2019}$. A smaller $\beta_{1,2020}$ is the result of a lower growth rate of M2.

Table 6. Prediction of 2019 yield curve based on NS model using monthly data from 2002 to 2018.

\begin{tabular}{ccccccccccc}
\hline$\tau$ & 1 & 2 & 3 & 6 & 9 & 12 & 24 & 36 & 48 \\
Predicted yield rate & 2.358 & 2.377 & 2.395 & 2.450 & 2.503 & 2.555 & 2.747 & 2.917 & 3.065 \\
Absolute prediction error & 0.243 & 0.173 & 0.132 & 0.094 & 0.085 & 0.095 & 0.091 & 0.101 & 0.133 \\
$\tau$ & 60 & 72 & 84 & 96 & 108 & 120 & 180 & 240 & 360 \\
Predicted yield rate & 3.194 & 3.306 & 3.404 & 3.488 & 3.562 & 3.626 & 3.846 & 3.969 & 4.097 \\
Absolute prediction error & 0.169 & 0.168 & 0.215 & 0.301 & 0.383 & 0.446 & 0.366 & 0.454 & 0.307 \\
\hline
\end{tabular}

Table 7. Prediction of 2019 yield curve based on VAR-NS model using monthly data from 2002 to 2018.

\begin{tabular}{ccccccccccc}
\hline$\tau$ & 1 & 2 & 3 & 6 & 9 & 12 & 24 & 36 & 48 \\
Predicted yield rate & 2.422 & 2.435 & 2.447 & 2.483 & 2.519 & 2.555 & 2.692 & 2.818 & 2.932 \\
Absolute prediction error & 0.313 & 0.224 & 0.167 & 0.094 & 0.080 & 0.091 & 0.094 & 0.094 & 0.097 \\
$\tau$ & 60 & 72 & 84 & 96 & 108 & 120 & 180 & 240 & 360 \\
Predicted yield rate & 3.033 & 3.123 & 3.202 & 3.271 & 3.332 & 3.385 & 3.571 & 3.676 & 3.785 \\
Absolute prediction error & 0.102 & 0.105 & 0.113 & 0.131 & 0.173 & 0.212 & 0.129 & 0.175 & 0.120 \\
\hline
\end{tabular}


Table 8. Prediction of parameters in VAR(Macro)-NS model using monthly data from 2002 to 2019.

\begin{tabular}{cccc}
\hline & $\beta_{1}$ & $\beta_{2}$ & $\beta_{3}$ \\
\hline $2020-01$ & 3.823 & -1.530 & -0.438 \\
$2020-02$ & 3.908 & -1.518 & -0.510 \\
$2020-03$ & 3.938 & -1.497 & -0.341 \\
$2020-04$ & 4.037 & -1.477 & -0.301 \\
$2020-05$ & 4.045 & -1.456 & -0.354 \\
$2020-06$ & 4.010 & -1.430 & -0.427 \\
$2020-07$ & 4.018 & -1.412 & -0.555 \\
$2020-08$ & 4.064 & -1.408 & -0.785 \\
$2020-09$ & 4.063 & -1.412 & -0.875 \\
$2020-10$ & 4.207 & -1.414 & -0.845 \\
$2020-11$ & 4.252 & -1.408 & -1.095 \\
$2020-12$ & 4.279 & -1.398 & -1.034 \\
\hline
\end{tabular}

Table 9. Prediction of 2020 yield curve based on VAR(Macro)-NS model using monthly data from 2002 to 2019.

\begin{tabular}{cccccccccc}
\hline$\tau$ & 1 & 2 & 3 & 6 & 9 & 12 & 24 & 36 & 48 \\
Predicted yield rate & 2.619 & 2.632 & 2.644 & 2.680 & 2.715 & 2.750 & 2.876 & 2.998 & 3.103 \\
$\tau$ & 60 & 72 & 84 & 96 & 108 & 120 & 180 & 240 & 360 \\
Predicted yield rate & 3.195 & 3.276 & 3.347 & 3.409 & 3.463 & 3.510 & 3.674 & 3.766 & 3.862 \\
\hline
\end{tabular}

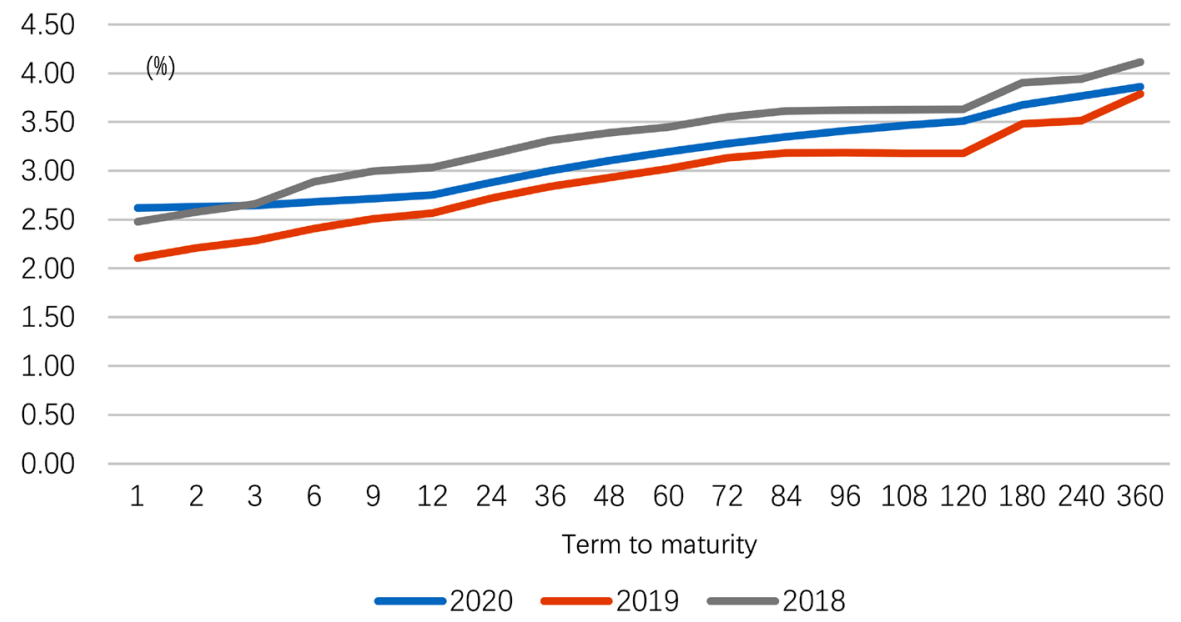

Figure 8. Treasury bond yield curve of 2018, 2019 and 2020e.

\section{Conclusion}

We settle down two main problems in the essay. First, we find that, although interest rate used to be incompletely regulated before 2014 in China, Nelson-Siegel model still shows excellent fitting efficiency. Second, we check whether adding 
macroeconomic variables to Nelson-Siegel model can enhance fitting efficiency. The essay compares three models including VAR (Macro)-NS model, simple NS model and VAR-NS model without macro variables, and finds that VAR (Macro)-NS model performs better than the others.

\section{Conflicts of Interest}

The author declares no conflicts of interest regarding the publication of this paper.

\section{References}

[1] Bank for International Settlement. Monetary and Economic Department (2005) Zero-Coupon Yield Curves: Technical Documentation. https://doi.org/10.2139/ssrn.1188514

[2] Diebold, F.X. and Li, C. (2006) Forecasting the Term Structure of Government Bond Yields. Journal of Econometrics, 130, 337-364. https://doi.org/10.1016/j.jeconom.2005.03.005

[3] Christensen, J.H., Diebold, F.X. and Rudebusch, G.D. (2009) An Arbitrage-Free Generalized Nelson-Siegel Term Structure Model. https://doi.org/10.3386/w14463

[4] Koopman, S.J., Mallee, M.I. and Van der Wel, M. (2010) Analyzing the Term Structure of Interest Rates Using the Dynamic Nelson-Siegel Model with Time-Varying Parameters. Journal of Business \& Economic Statistics, 28, 329-343. https://doi.org/10.1198/jbes.2009.07295

[5] Shen, G.X. (2010) Test of Expectation Theory of the Term Structure in China Interbank Market. Shanghai Journal of Economics, No. 4, 39-44.

[6] Nelson, C.R. and Siegel, A.F. (1987) Parsimonious Modeling of Yield Curves. Journal of Business, 60, 473-489. https://doi.org/10.1086/296409

[7] Shen, G.X. (2017) Factor Transformation of Dynamic Term Structure Model with appliY612cations. Statistical Research, 34, 119-128.

[8] Ang, A. and Piazzesi, M. (2003) A No-Arbitrage Vector Auto-Regression of Term Structure Dynamics with Macroeconomic and Latent Variables. Journal of Monetary Economics, 50, 745-787. https://doi.org/10.1016/S0304-3932(03)00032-1

[9] Bikbov, R. and Chernov, M. (2010) No-Arbitrage Macroeconomic Determinants of the Yield Curve. Journal of Econometrics, 159, 166-182. https://doi.org/10.1016/j.jeconom.2010.05.004

[10] Hordahl, P., Tristani, O. and Vestin, D. (2006) A Joint Econometric Model of Macroeconomic and Term-Structure Dynamics. Journal of Economics, 131, 405-444. https://doi.org/10.1016/j.jeconom.2005.01.012

[11] Coroneo, L., Giannone, D. and Modugno, M. (2016) Unspanned Macroeconomic Factors in the Yield Curve. Journal of Business \& Economic Statistics, 34, 472-485. https://doi.org/10.1080/07350015.2015.1052456

[12] Guidolin, M. and Thornton, D.L. (2018) Predictions of Short-Term Rates and the Expectations Hypothesis. International Journal of Forecasting, 34, 636-664. https://doi.org/10.1016/j.ijforecast.2018.03.006

[13] Qiang, J., Hou, X. and Fan, L.Z. (2018) Base Interest Rate, Expected Inflation and the Mechanism for Determining the Term Structure of Market Interest Rate in China. Economic Research Journal, 53, 92-107. 